\title{
El índice de masa corporal predice mortalidad
}

Body Mass Index and Mortality in a prospective cohort of U.S adults.

Calle E; Thun M; Petrelli J; NEJM 1999,october 7; 341: 1097-1105

\section{Objetivos}

Evaluar la asociación entre Indice de Masa Corporal (IMC) y mortalidad por todas las causas y por enfermedad cardiovascular (ECV). Evaluar la influencia de la edad, la raza, el sexo, el tabaquismo y las enfermedades concomitantes en la relación IMC-Mortalidad.

\section{Diseño}

Cohorte prospectiva* con 14 años de seguimiento.

\section{Lugar}

Estados Unidos (50 estados), distrito de Columbia y Puerto Rico.

\section{Pacientes}

457.785 hombres y 588.369 mujeres, mayores de 30 años y con una edad promedio de 57, fueron seleccionados del Cancer Preventive Study II (un estudio de mortalidad llevado a cabo por la American Cancer Society). Los participantes habían completado un cuestionario en 1982 y se registraron todas las muertes ocurridas hasta diciembre de 1996.

\section{Evaluación de factores pronósticos}

IMC: se estratificó en categorías desde menor de 18.5 hasta mayor o igual a 40.0. Se establecieron 4 subgrupos de riesgo en base a la presencia o ausencia de tabaquismo (TBQ) y enfermedades concomitamtes [EC] (cáncer, ACV, ECV, respiratoria, cualquier otra enfermedad y perdida de peso de $4.5 \mathrm{~kg}$ en el año previo).

\section{Medición de resultados principales}

Las tasas de mortalidad se expresaron en riesgo relativo comparando cada categoría de IMC con la de referencia: 23.5-24.9. Para ca-

da uno de los cuatro subgrupos se midió asociación entre IMC y mortalidad de acuerdo al sexo.

\section{Resultados principales}

En los 14 años de seguimiento se produjeron 201.622 muertes, $20,1 \%$ de la población en estudio.

El riesgo absoluto* de muerte ajustado por edad varió sustancialmente entre los cuatro subgrupos, observándose la mayor mortalidad en el subgrupo TBQ con EC, y en orden decreciente para los grupos TBO sin EC, No TBQ con EC, y No TBQ sin EC. En el grupo más sano (no TBQ ni EC) se observó que el RR de muerte para la categoría de IMC máximo, fue 2.68 (IC 95\% 1.76-4.08) en hombres y 1.89 (IC $95 \% \cdot 1.26-2.21$ ) en mujeres. También hubo más mortalidad en los más delgados: IMC mínimo RR 1.28 (IC 95\% 1.04-1,58) en hombres y 1.36 (IC 95\% 1.26-1.48) en mujeres.

Un alto IMC fue predictor de muerte por ECV especialmente en hombres, donde se observó un RR para la categoría de máximo IMC de 2.90 (IC 95\% 2.37-3.56). El riesgo de muerte por cáncer fue entre un 40 y $80 \%$ mayor en la categoría máxima de IMC y no hubo aumento de riesgo en las personas más delgadas. La mortalidad fue mayor en los obesos de todas las edades, pero el impacto de la misma fue de menor importancia en personas de mayor edad.

\section{Conclusiones}

El riesgo de muerte por todas las causas, cardiovascular, cáncer, y otras enfermedades se incrementó en forma progresiva a medida que aumentaba el grado de obesidad, para ambos sexos y en todas las edades.

\section{COMENTARIO}

Aunque este estudio no fue diseñado para establecer si la obesidad es un factor independiente* de muerte queda claro que la poblacion obesa tiene mas riesgo de morir que la poblacion no obesa. El hallazgo de un RR de muerte mayor en los obesos mas sanos (no TBQ sin EC) hace razonable atribuir dicho riesgo a patologías asociadas a la obesidad. Como es sabido, la prevalencia de diabetes y de hipertensión arterial es mucho mayor en los obesos (alrededor de 3 veces mayor) con el consiguiente aumento de riesgo de ECV. También aumenta el riesgo de desarrollar dislipemia, ciertos cánceres (como endometrio, ovario, mama, colon) depresión, osteoartrosis, etc.

Algunas causas probables de sesgos en este estudio, son: 1) la encuesta no fue diseñada específicamente para este trabajo, 2) al ser información autoreferida, el desconocimiento de enfermedades que padecieran pudo haber generado subregistro asi como los pesos y las tallas reportados (con la conocida sobreestimación de la talla y subestimación del peso).

Sin embargo los resultados fueron similares a otros estudios. ${ }^{1-2} \mathrm{El}$ presente trabajo junto con el reportado por Stevens, ${ }^{3}$ son los dos trabajos más grandes que analizaron IMC y mortalidad hasta el pre-

\section{*Ver glosario}

\section{Dra. Verónica Schoj}

Unidad de Medicina Familiar y Preventiva. Hospital Italiano de Buenos Aires. sente y arrojan resultados semejantes.

Las armas terapéuticas más efectivas en obesidad siguen siendo un adecuado plan de alimentación y un programa de actividad física con un intenso trabajo sobre los cambios de conductas. Sin embargo los resultados siguen siendo desalentadores.

Las contribuciones, que para nuestra practica clínica, podrían inferirse del presente trabajo son:

1) Entendiendo el mayor riesgo de muerte de la poblacion obesa y dada la altísima tasa de fracaso terapéutico, sería recomendable abordar, simultáneamente con el programa de descenso de peso, el tratamiento de las enfermedades asociadas.

2) Conociendo cuales son las patologías asociadas que ponen en riesgo a dicha población, aumentar el índice de sospecha de las mismas para detectarlas e iniciar su tratamiento tempranamente.

3) Su enorme prevalencia, el riesgo que conlleva y su difícil tratamiento, sugieren que la Prevención Primaria sería la herramienta más costoefectiva que podríamos desempeñar los médicos de atención primaria. Y nuestro lugar es de privilegio para ello a través de la estimulación de patrones alimentarios más saludables y del consejo sobre la realización de actividad física sistemática.

\section{Referencias}

1. Min L.; Manson J.; Hennekens C.; JAMA Dec 15,1993; 270: 2823-2828

2. Manson J.; Willett W.; Stampfer M. NEJM 1995;333:677-685.

3. Stevens J.; Cai J., Pamuk E.; NEJM 1998;338:1-7. 